\title{
A Biomimetic $\mathrm{Sn}_{4} \mathrm{P}_{3}$ Anchored on Carbon
}

\section{Nanotubes as an Anode for High-Performance}

\section{Sodium-Ion Batteries}

Lingbing Ran,,$^{\dagger}$ Bin Luo, ${ }^{\#}$ Ian R. Gentle,, Tongen Lin, ${ }^{*}$ Qiang Sun, ${ }^{\dagger}$ Ming Li, ${ }^{\dagger}$ Md Masud Rana, ${ }^{\dagger}$ Lianzhou Wang ${ }^{\#, *}$ and Ruth Knibbe $e^{\dagger, *}$

†School of Mechanical and Mining Engineering, The University of Queensland, Brisbane, QLD 4072, Australia.

\#Australian Institute for Bioengineering and Nanotechnology (AIBN), The University of Queensland, Brisbane, QLD 4072, Australia.

${ }^{\S}$ School of Chemistry and Molecular Biosciences, The University of Queensland, Brisbane, QLD 4072, Australia

*Corresponding author. Email: ruth.knibbe@uq.edu.au, l.wang@uq.edu.au. 
Figure captions

Figure S1. TGA of pure $\mathrm{Sn}_{4} \mathrm{P}_{3}$.

Figure S2. TGA of $\mathrm{SnO}_{2} @ \mathrm{CNT} / \mathrm{C}$.

Figure S3. SEM of pure $\mathrm{Sn}_{4} \mathrm{P}_{3}$.

Figure S4. XPS spectra scans survey of $\mathrm{Sn}_{4} \mathrm{P}_{3} @ \mathrm{CNT} / \mathrm{C}$.

Figure S5. a) TEM of $\mathrm{Sn}_{4} \mathrm{P}_{3} @ \mathrm{CNT} / \mathrm{C}$ and b) Particle size distribution of $\mathrm{Sn}_{4} \mathrm{P}_{3}$.

Figure S6. SEM of $\mathrm{Sn}_{4} \mathrm{P}_{3} @ \mathrm{CNT} / \mathrm{C}$ after 500 cycles.

Figure S7. a) XRD of $\mathrm{Na}_{3} \mathrm{~V}_{2}\left(\mathrm{PO}_{4}\right)_{3} / \mathrm{C}$, b) Charge/discharge curves of $\mathrm{Na}_{3} \mathrm{~V}_{2}\left(\mathrm{PO}_{4}\right)_{3} / \mathrm{C}$ $(\mathrm{NVP} / \mathrm{C})$ and $\mathrm{Sn}_{4} \mathrm{P}_{3} @ \mathrm{CNT} / \mathrm{C}$ in half cells, c) Cycling stability of $\mathrm{Na}_{3} \mathrm{~V}_{2}\left(\mathrm{PO}_{4}\right)_{3} / \mathrm{C}$ at a current of $23.4 \mathrm{~mA} \mathrm{~g}^{-1}$ and d) Cycling performance and Coulombic efficiency of coin-type $\mathrm{NVP} / \mathrm{C} / \mathrm{Sn}_{4} \mathrm{P}_{3} @ \mathrm{CNT} / \mathrm{C}$ full cell at $0.2 \mathrm{C}$.

Figure S8. Energy density comparison of different sodium battery systems.

Figure S9. EDS mapping images of $\mathrm{Sn}_{4} \mathrm{P}_{3} @ \mathrm{CNT} / \mathrm{C}$ at a) $0.01 \mathrm{~V}$ (end of first sodiation cycle) and b) $2.0 \mathrm{~V}$ (end of first desodiation cycle).

Figure S10. SEM images of $\mathrm{Sn}_{4} \mathrm{P}_{3}$ electrode: a) before cycling and b) after 500 cycles.

Table S1. Recent progress on the electrochemical performance on tin phosphide for SIBs.

Table S2. Resistance $\left(\mathrm{R}_{\Omega}, \mathrm{R}_{\mathrm{s}}, \mathrm{R}_{\mathrm{ct}}, \mathrm{Z}_{\mathrm{w}}\right)$ for $\mathrm{Sn}_{4} \mathrm{P}_{3} @ \mathrm{CNT} / \mathrm{C}$ at different charge/discharge state.

Table S3. Resistance $\left(\mathrm{R}_{\Omega}, \mathrm{R}_{\mathrm{s}}, \mathrm{R}_{\mathrm{ct}}, \mathrm{Z}_{\mathrm{w}}\right)$ for pure $\mathrm{Sn}_{4} \mathrm{P}_{3}$ and $\mathrm{Sn}_{4} \mathrm{P}_{3} @ \mathrm{CNT} / \mathrm{C}$ at different state.

Table S4. Resistance $\left(\mathrm{R}_{\Omega}, \mathrm{R}_{\mathrm{s}}, \mathrm{R}_{\mathrm{ct}}, \mathrm{Z}_{\mathrm{w}}\right)$ for $\mathrm{Sn}_{4} \mathrm{P}_{3} @ \mathrm{CNT} / \mathrm{C}$ at different cycles. 


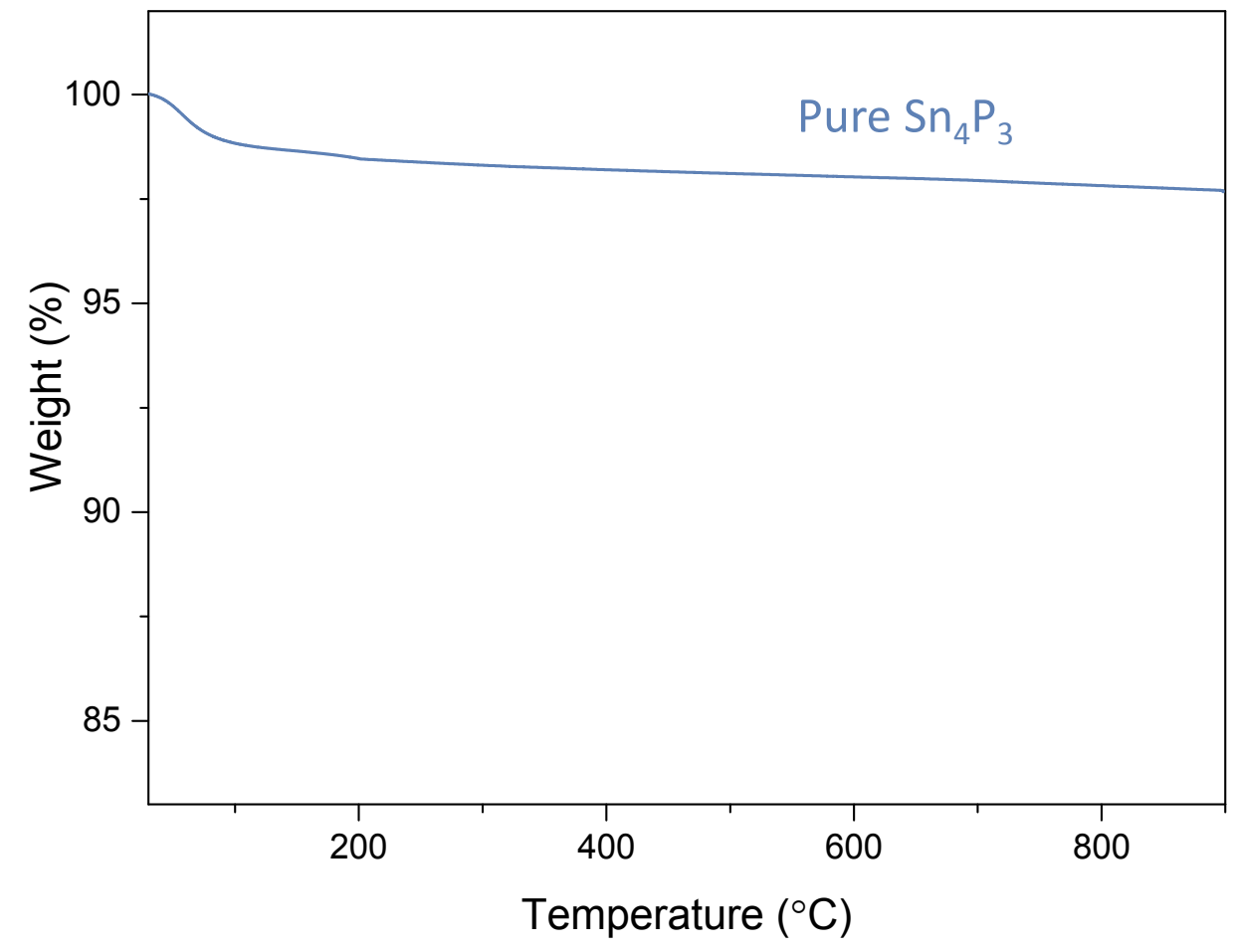

Figure S1. TGA of pure $\mathrm{Sn}_{4} \mathrm{P}_{3}$.

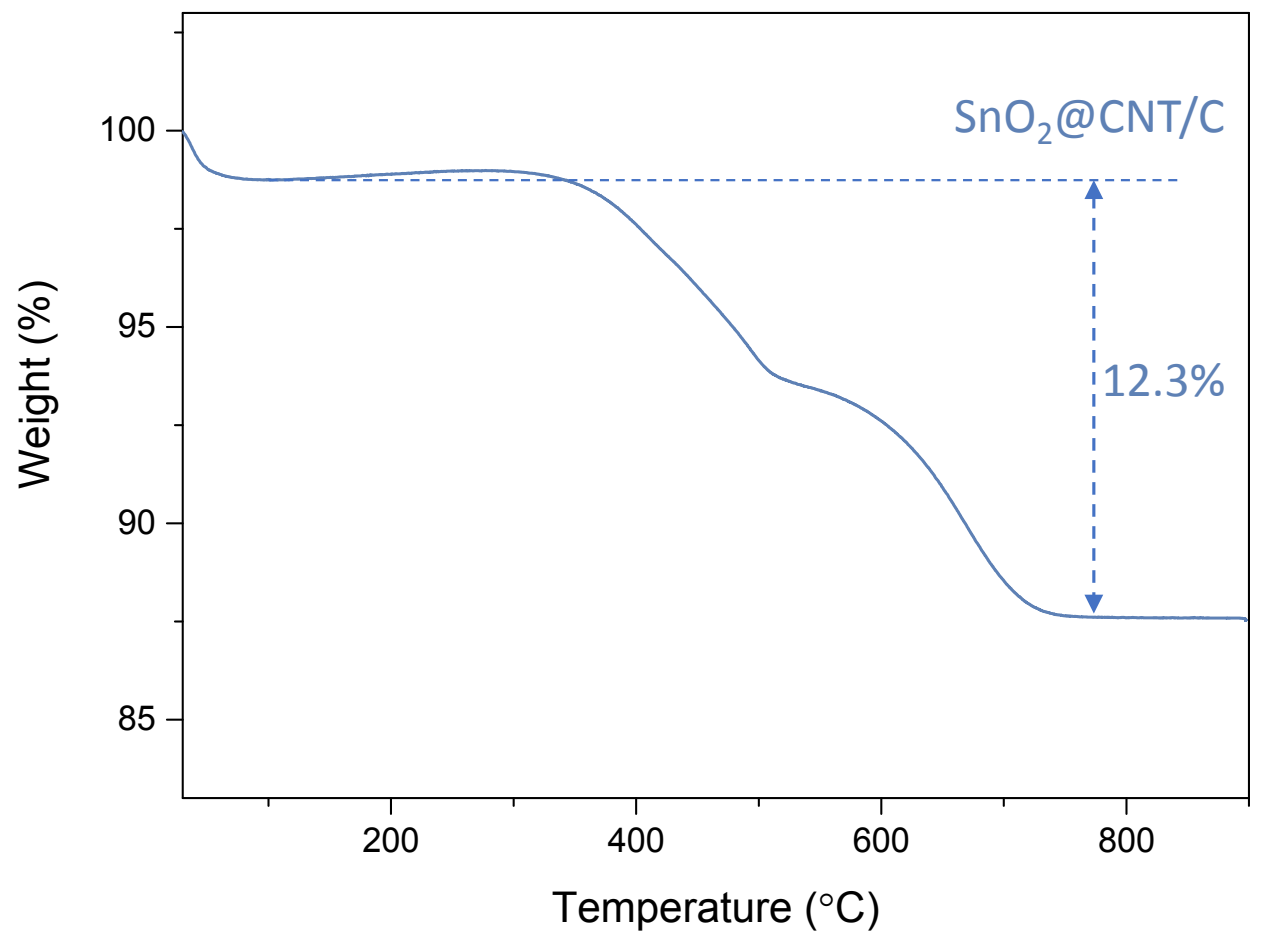

Figure S2. TGA of $\mathrm{SnO}_{2} @ \mathrm{CNT} / \mathrm{C}$. 


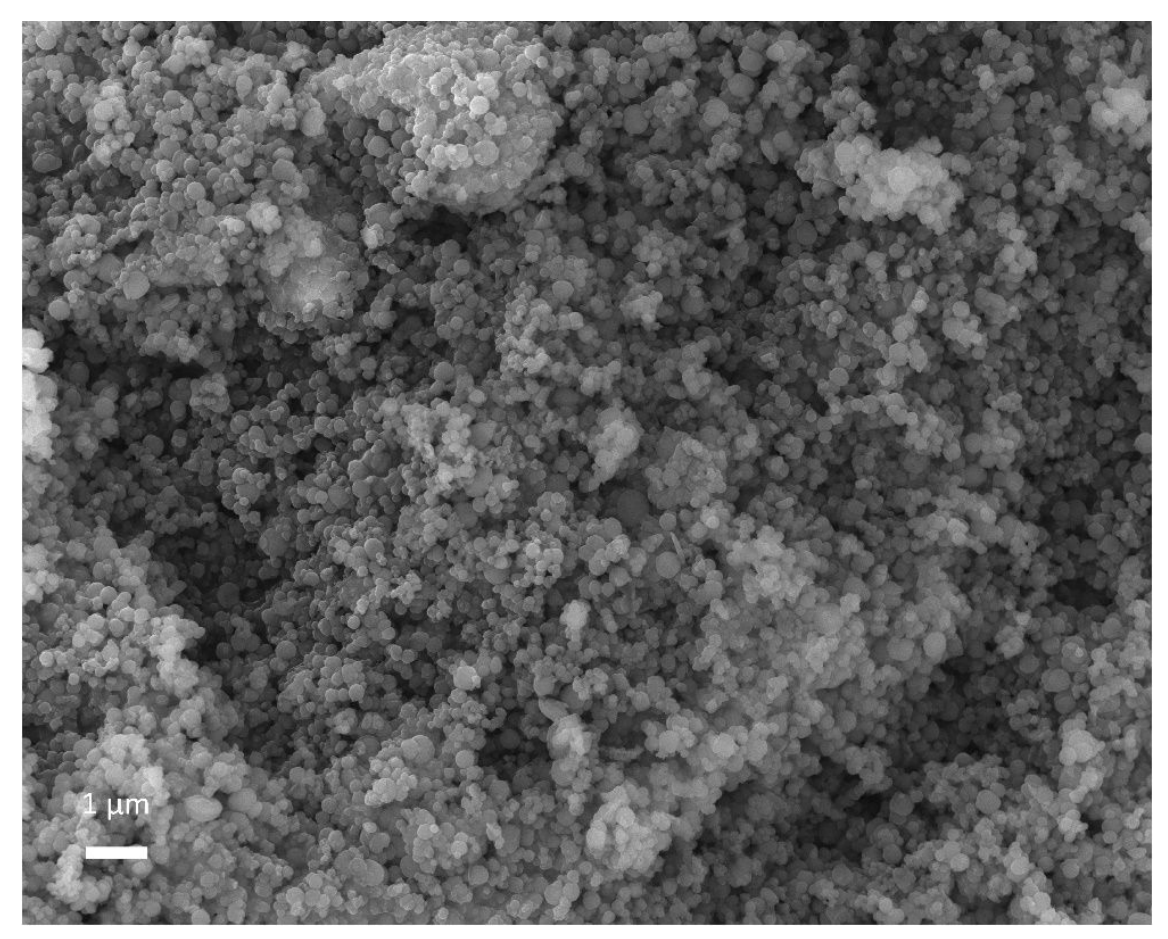

Figure S3. SEM of pure $\mathrm{Sn}_{4} \mathrm{P}_{3}$.

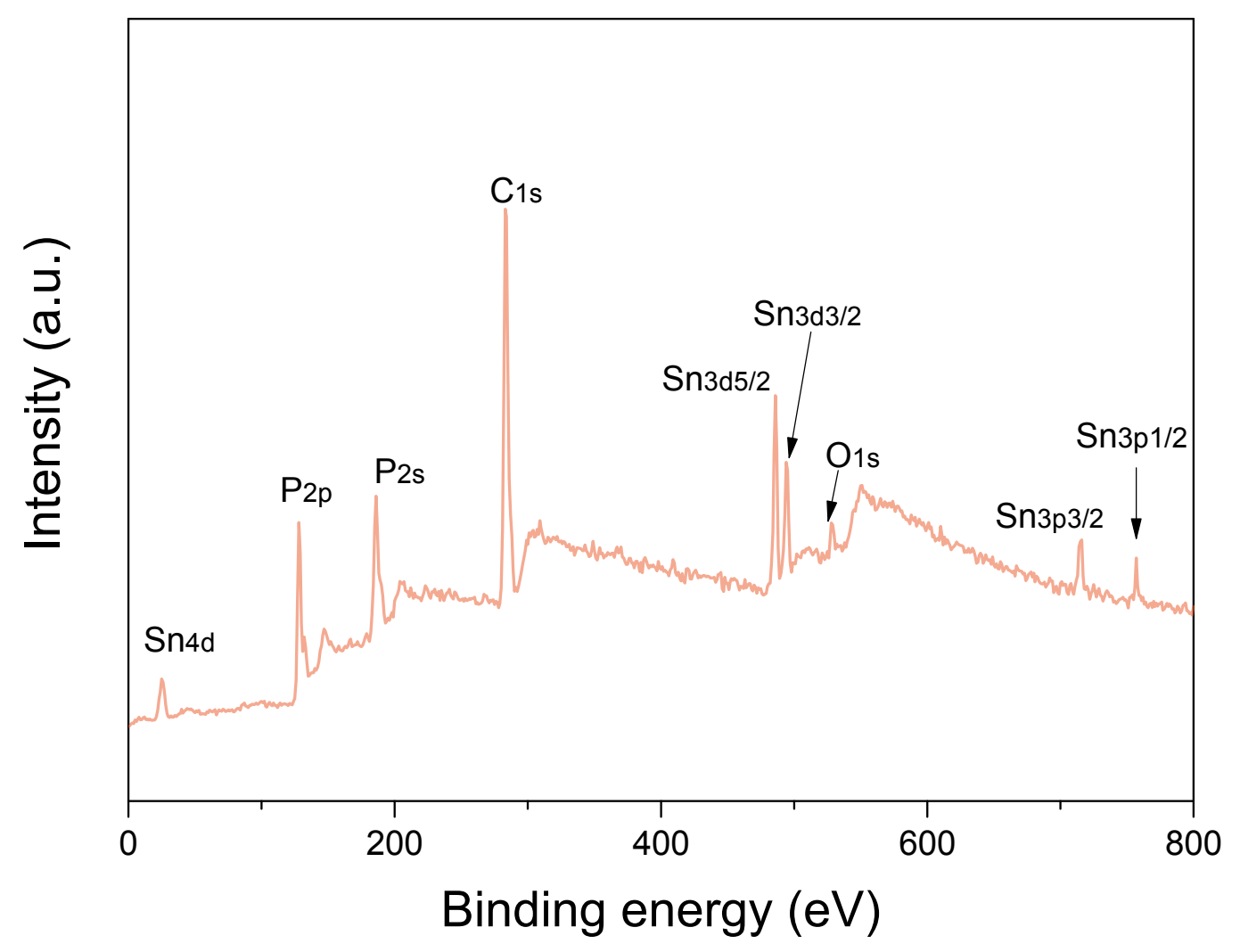

Figure S4. XPS spectra scans survey of $\mathrm{Sn}_{4} \mathrm{P}_{3} @ \mathrm{CNT} / \mathrm{C}$. 
a)

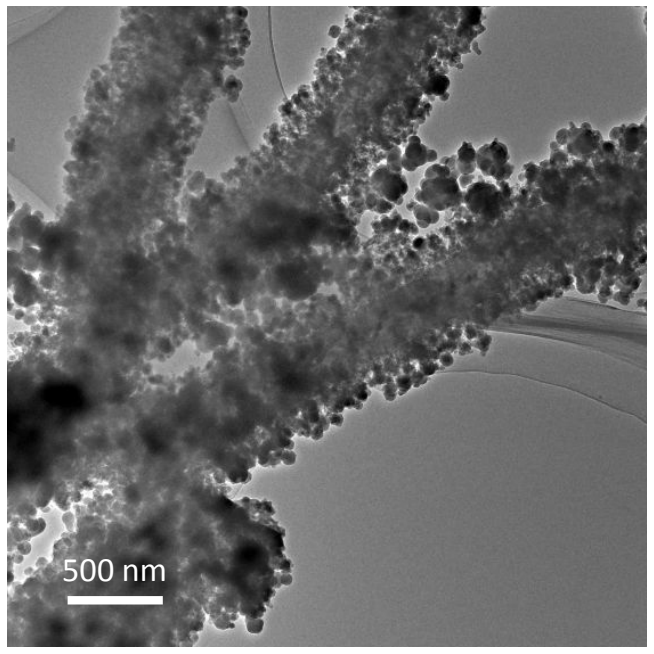

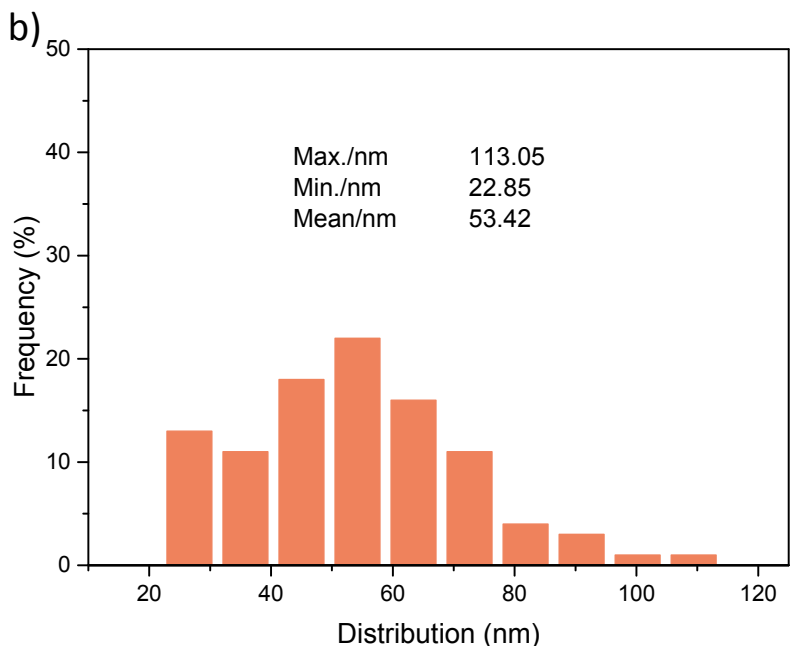

Figure S5. a) TEM of $\mathrm{Sn}_{4} \mathrm{P}_{3} @ \mathrm{CNT} / \mathrm{C}$ and b) Particle size distribution of $\mathrm{Sn}_{4} \mathrm{P}_{3}$.

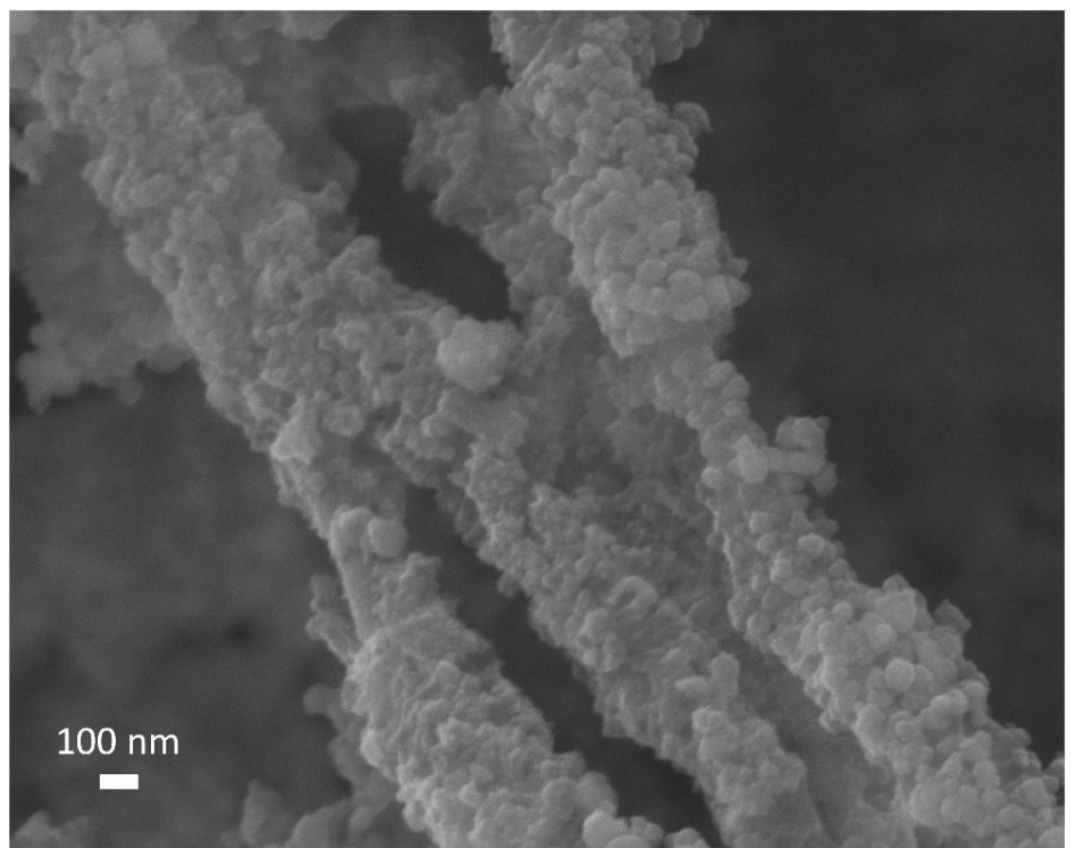

Figure S6. SEM of $\mathrm{Sn}_{4} \mathrm{P}_{3} @ \mathrm{CNT} / \mathrm{C}$ after 500 cycles. 

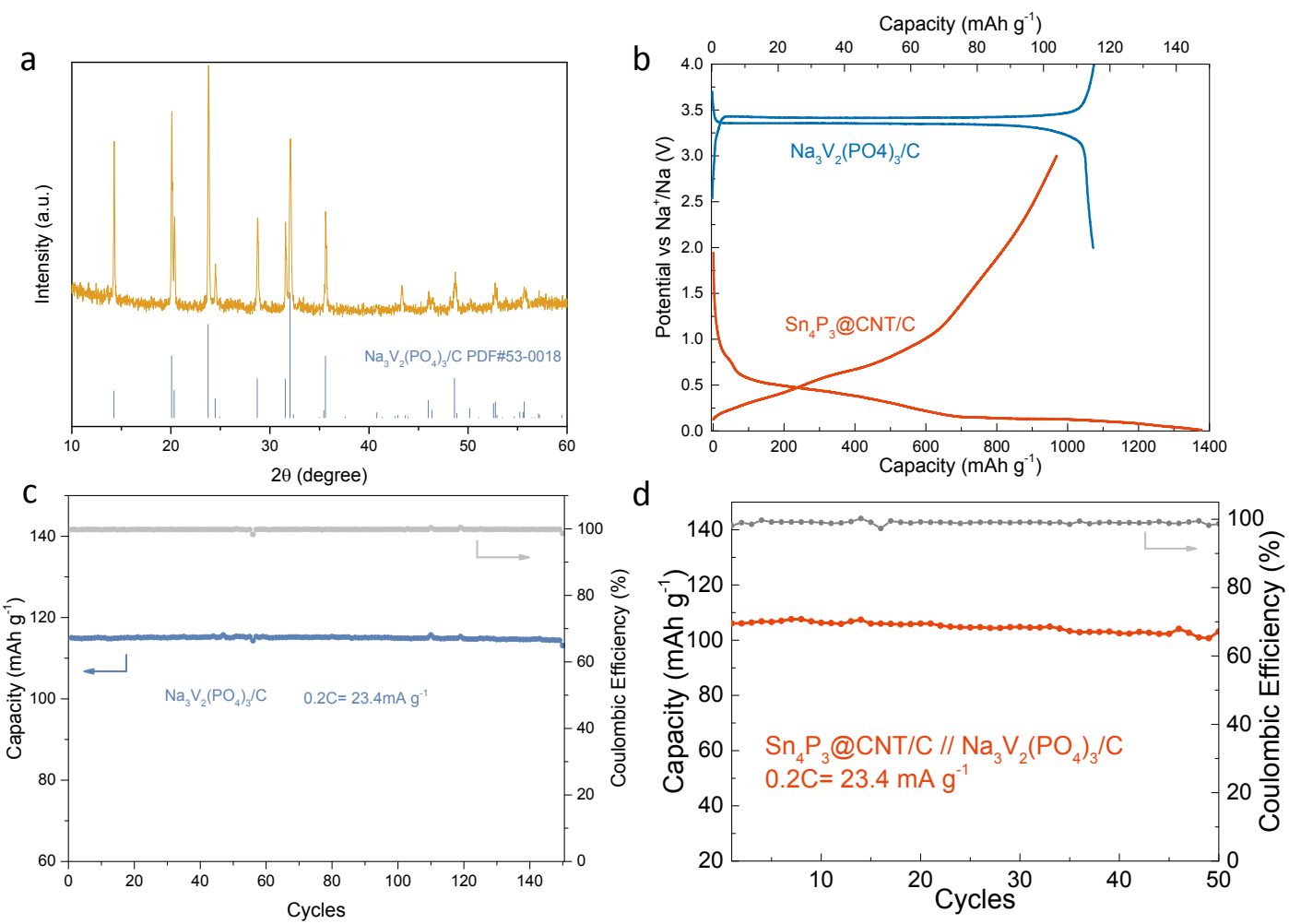

Figure S7. a) XRD of $\mathrm{Na}_{3} \mathrm{~V}_{2}\left(\mathrm{PO}_{4}\right)_{3} / \mathrm{C}$, b) Charge/discharge curves of $\mathrm{Na}_{3} \mathrm{~V}_{2}\left(\mathrm{PO}_{4}\right)_{3} / \mathrm{C}$ $(\mathrm{NVP} / \mathrm{C})$ and $\mathrm{Sn}_{4} \mathrm{P}_{3} @ \mathrm{CNT} / \mathrm{C}$ in half cells, c) Cycling stability of $\mathrm{Na}_{3} \mathrm{~V}_{2}\left(\mathrm{PO}_{4}\right)_{3} / \mathrm{C}$ at a current of $23.4 \mathrm{~mA} \mathrm{~g}^{-1}$ and d) Cycling performance and Coulombic efficiency of coin-type $\mathrm{NVP} / \mathrm{C} / / \mathrm{Sn}_{4} \mathrm{P}_{3} @ \mathrm{CNT} / \mathrm{C}$ full cell at 0.2C.

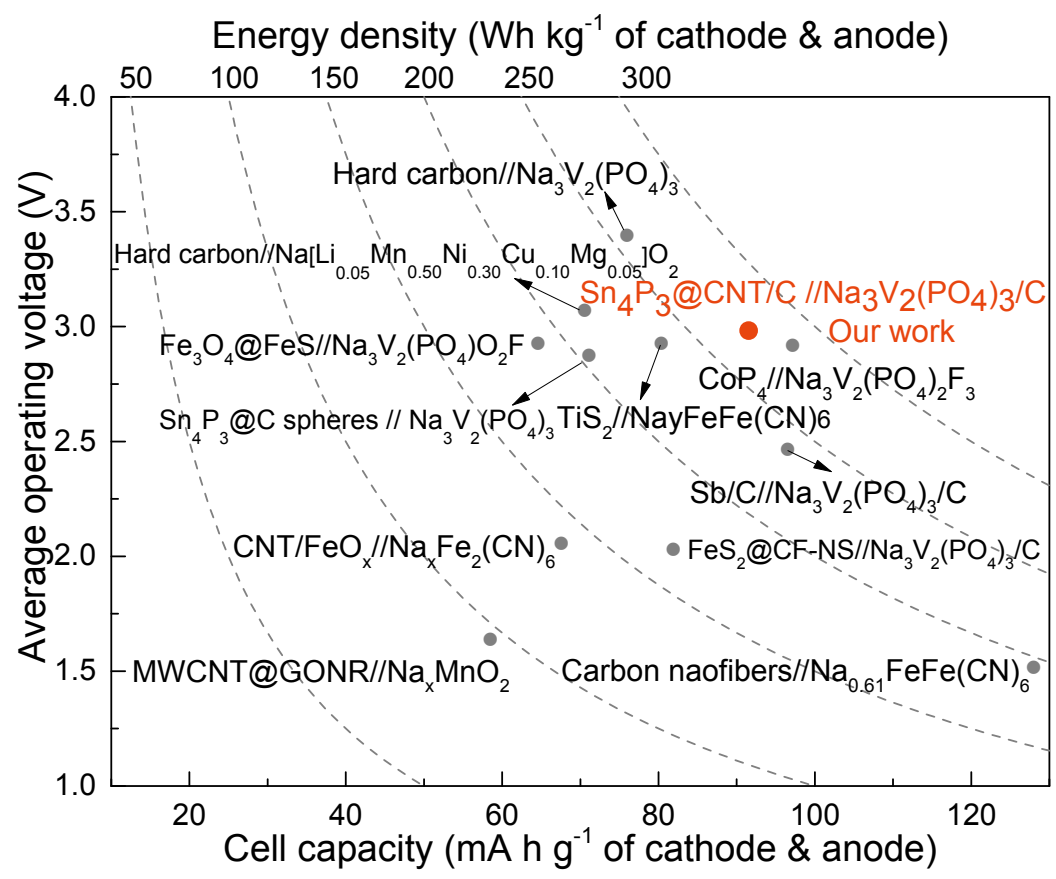

Figure S8. Energy density comparison of different sodium battery systems. 

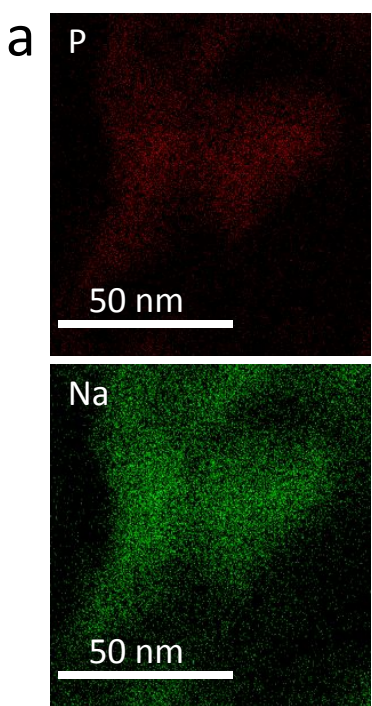
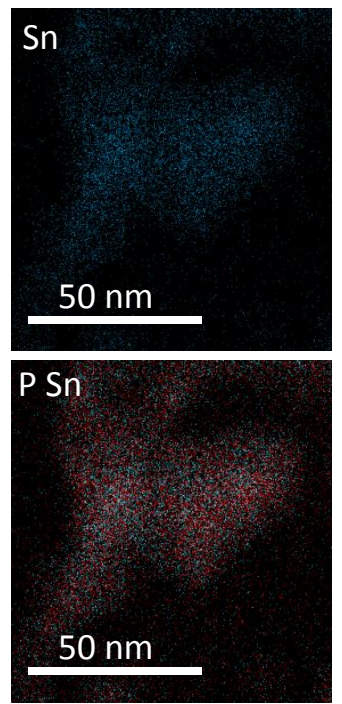
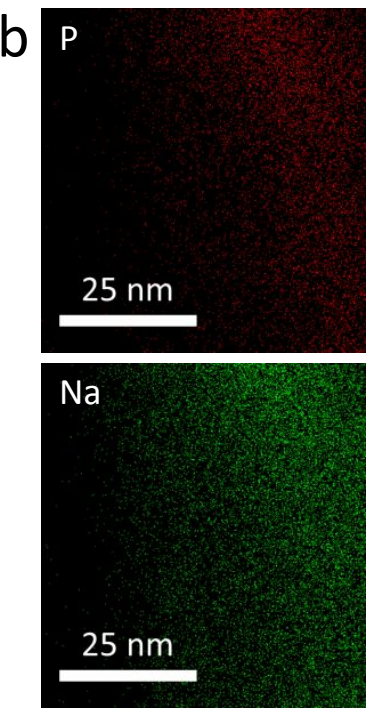
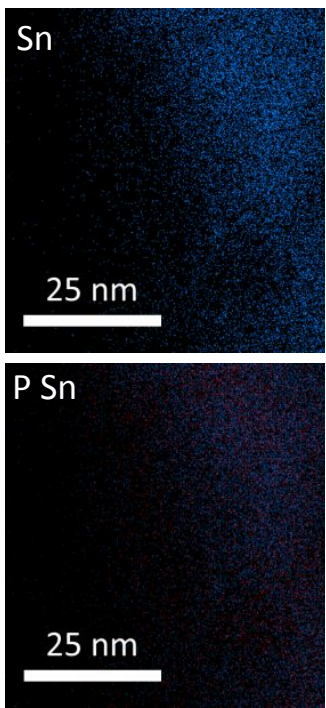

Figure S9. EDS mapping images of $\mathrm{Sn}_{4} \mathrm{P}_{3} @ \mathrm{CNT} / \mathrm{C}$ at a) $0.01 \mathrm{~V}$ (end of first sodiation cycle) and b) $2.0 \mathrm{~V}$ (end of first desodiation cycle).
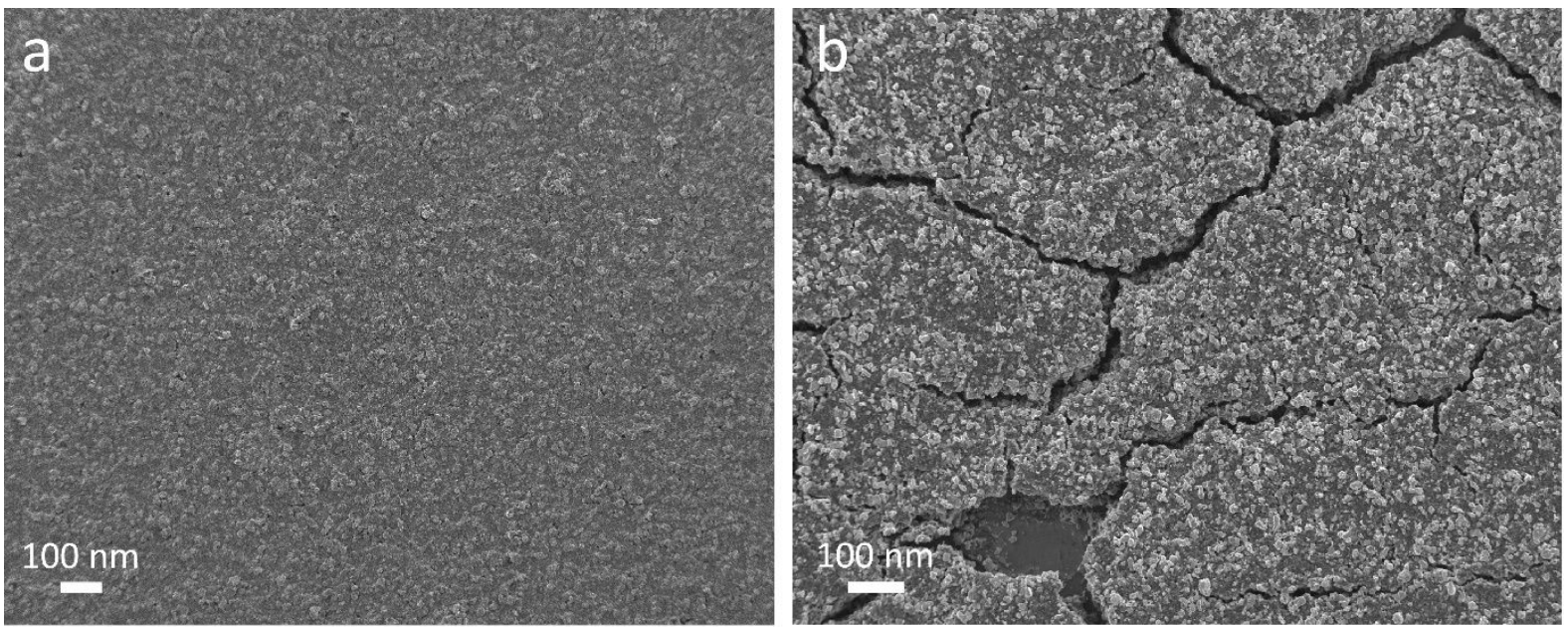

Figure S10. SEM images of $\mathrm{Sn}_{4} \mathrm{P}_{3}$ electrode: a) before cycling and b) after 500 cycles. 
Table S1. Recent progress on the electrochemical performance on tin phosphide for SIBs.

\begin{tabular}{|c|c|c|c|c|c|}
\hline $\begin{array}{c}\text { Materials } \\
\text { description }\end{array}$ & Electrolyte & $\begin{array}{c}\text { Initial } \\
\text { Coulombic } \\
(\%)\end{array}$ & Cycling data ${ }^{\text {a) }}$ & $\begin{array}{c}\text { Rate } \\
\text { capability } \\
\text { b) }\end{array}$ & Reference \\
\hline $\begin{array}{l}\mathrm{Sn}_{4} \mathrm{P}_{3} \\
\text { Microspheres }\end{array}$ & $\begin{array}{c}1 \mathrm{M} \mathrm{NaClO}_{4} \text { in } \\
\mathrm{DMC} / \mathrm{EC}(\mathrm{v} / \mathrm{v} \\
=1: 1) \text { with } 5 \% \\
\text { FEC }\end{array}$ & 64 & $420 / 300 / 0.2 C$ & $175 / 2 \mathrm{C}$ & 1 \\
\hline $\mathrm{Sn}_{4} \mathrm{P}_{3}$ & $\begin{array}{c}1 \mathrm{M} \mathrm{NaClO}_{4} \text { in } \\
\mathrm{PC} / \mathrm{FEC}(\mathrm{v} / \mathrm{v}= \\
95: 5)\end{array}$ & 85 & $\approx 450 / 100 / 0.1 \mathrm{C}$ & / & 2 \\
\hline $\begin{array}{l}\mathrm{Sn}_{4} \mathrm{P}_{3}-\mathrm{C} \\
\text { nanospheres }\end{array}$ & $\begin{array}{c}1 \mathrm{M} \mathrm{NaClO}_{4} \text { in } \\
\mathrm{EC} / \mathrm{DMC}(\mathrm{v} / \mathrm{v} \\
=1: 1) \text { with } 5 \% \\
\text { FEC }\end{array}$ & 60 & $650 / 50 / 0.2 \mathrm{C}$ & $420 / 2 \mathrm{C}$ & 3 \\
\hline $\mathrm{Sn}_{4} \mathrm{P}_{3} / \mathrm{rGO}$ & $\begin{array}{c}1 \mathrm{M} \mathrm{NaClO}_{4} \text { in } \\
\mathrm{PC} / \mathrm{FEC}(\mathrm{v} / \mathrm{v}= \\
95: 5)\end{array}$ & 47 & $656 / 100 / 0.1 \mathrm{C}$ & $391 / 2 \mathrm{C}$ & 4 \\
\hline $\begin{array}{l}\mathrm{Sn}_{4} \mathrm{P}_{3} @ \mathrm{C} \\
\text { spheres }\end{array}$ & $\begin{array}{c}1 \mathrm{M} \mathrm{NaClO}_{4} \text { in } \\
\mathrm{DME}^{2}\end{array}$ & 90 & $700 / 120 / 0.1 \mathrm{C}$ & / & 5 \\
\hline $\begin{array}{l}\text { Yolk-shell } \\
\mathrm{Sn}_{4} \mathrm{P}_{3} @ \mathrm{C} \\
\text { nanospheres }\end{array}$ & $\begin{array}{c}1 \mathrm{M} \mathrm{NaClO}_{4} \text { in } \\
\mathrm{PC} / \mathrm{FEC}(\mathrm{v} / \mathrm{v}= \\
95: 5)\end{array}$ & $\approx 42$ & $515 / 50 / 0.1 \mathrm{C}$ & $421 / 3 C$ & 6 \\
\hline $\mathrm{Sn}_{4} \mathrm{P}_{3} / \mathrm{rGO}$ & $\begin{array}{c}1 \mathrm{M} \\
\text { NaFSA/Py13- } \\
\text { FSA. }\end{array}$ & 83.5 & $800 / 100 / 0.05 \mathrm{C}$ & / & 7 \\
\hline $\mathrm{Sn}_{4} \mathrm{P}_{3}$ & $\begin{array}{c}1 \mathrm{M} \mathrm{NaClO}_{4} \text { in } \\
\mathrm{DEC} / \mathrm{EC}(\mathrm{v} / \mathrm{v}= \\
1: 1)\end{array}$ & / & $650 / 100 / 0.1 \mathrm{C}$ & / & 8 \\
\hline $\mathrm{Sn}_{4} \mathrm{P}_{3}$-graphene & $\begin{array}{c}1 \mathrm{M} \mathrm{NaClO}_{4} \text { in } \\
\mathrm{EC} / \mathrm{DMC}(\mathrm{v} / \mathrm{v} \\
=1: 1) \text { with } 5 \% \\
\text { FEC }\end{array}$ & 67.3 & $657 / 100 / 0.1 \mathrm{C}$ & $403 / 2 \mathrm{C}$ & 9 \\
\hline $\begin{array}{l}\text { Our work: } \\
\mathrm{Sn}_{4} \mathrm{P}_{3} @ \mathrm{CNT} / \mathrm{C}\end{array}$ & $\begin{array}{c}1 \mathrm{M} \mathrm{NaClO}_{4} \text { in } \\
\mathrm{DME}^{-}\end{array}$ & 85.2 & $742 / 150 / 0.2 \mathrm{C}$ & $449 / 2 \mathrm{C}$ & / \\
\hline
\end{tabular}

a)The cycling data are summarized as capacity/corresponding cycle number/corresponding current density. The specific capacity was calculated based on the weight of composite. The unit of capacity is $\mathrm{mA} \mathrm{h} \mathrm{g}^{-1}$. For all the data, $1 \mathrm{C}$ equals $1000 \mathrm{~mA} \mathrm{~g}^{-1}$.

b) The rate capability is summarized as capacity/corresponding current density. The specific capacity was calculated based on the weight of composite. The unit of capacity is $\mathrm{mA} \mathrm{h} \mathrm{g}^{-1}$. For all the data, $1 \mathrm{C}$ equals $1000 \mathrm{~mA} \mathrm{~g}^{-1}$. 
Table S2. Resistance $\left(\mathrm{R}_{\Omega}, \mathrm{R}_{\mathrm{s}}, \mathrm{R}_{\mathrm{ct}}, \mathrm{Z}_{\mathrm{w}}\right)$ for $\mathrm{Sn}_{4} \mathrm{P}_{3} @ \mathrm{CNT} / \mathrm{C}$ at different charge/discharge state.

\begin{tabular}{lcccc}
\hline \multicolumn{1}{c}{ Sample } & $\mathrm{R}_{\Omega}$ & $\mathrm{R}_{\mathrm{s}}(\Omega)$ & $\mathrm{R}_{\mathrm{ct}}(\Omega)$ & $\mathrm{Z}_{\mathrm{w}}\left(\mathrm{Ohm} . \mathrm{s}^{\wedge-1 / 2}\right)$ \\
\hline Pristine & 1.5 & 257.5 & 1025.0 & 659.2 \\
Discharge 0.25V & 2.3 & 203.6 & 554.6 & 194.6 \\
Discharge 0.01V & 2.2 & 99.4 & 196.0 & 65.6 \\
Charge 0.5V & 3.4 & 13.4 & 35.6 & 23.2 \\
Charge 2.0V & 2.0 & 8.5 & 3.4 & 35.7 \\
\hline
\end{tabular}

Table S3. Resistance $\left(\mathrm{R}_{\Omega}, \mathrm{R}_{\mathrm{s}}, \mathrm{R}_{\mathrm{ct}}, \mathrm{Z}_{\mathrm{w}}\right)$ for pure $\mathrm{Sn}_{4} \mathrm{P}_{3}$ and $\mathrm{Sn}_{4} \mathrm{P}_{3} @ \mathrm{CNT} / \mathrm{C}$ at different state.

\begin{tabular}{lcccl}
\hline \multicolumn{1}{c}{ Sample } & $\mathrm{R}_{\Omega}$ & $\mathrm{R}_{\mathrm{s}}(\Omega)$ & $\mathrm{R}_{\mathrm{ct}}(\Omega)$ & $\mathrm{Z}_{\mathrm{w}}\left(\mathrm{Ohm}_{\mathrm{s}} \mathrm{s}^{\wedge-1 / 2}\right)$ \\
\hline Pure $\mathrm{Sn}_{4} \mathrm{P}_{3}-1$ cycle & 2.3 & 287.2 & 357.2 & 57.4 \\
Pure $\mathrm{Sn}_{4} \mathrm{P}_{3}-500$ cycles & 2.8 & 339.6 & 495.5 & 44.3 \\
$\mathrm{Sn}_{4} \mathrm{P}_{3} @$ CNT/C- 1 cycle & 1.7 & 105.3 & 278.0 & 45.1 \\
$\mathrm{Sn}_{4} \mathrm{P}_{3} @$ CNT/C- 500 cycles & 1.4 & 160.1 & 112.3 & 97.8 \\
\hline
\end{tabular}

Table S4. Resistance $\left(\mathrm{R}_{\Omega}, \mathrm{R}_{\mathrm{s}}, \mathrm{R}_{\mathrm{ct}}, \mathrm{Z}_{\mathrm{w}}\right)$ for $\mathrm{Sn}_{4} \mathrm{P}_{3} @ \mathrm{CNT} / \mathrm{C}$ at different cycles.

\begin{tabular}{ccccc}
\hline Cycles & $\mathrm{R}_{\Omega}$ & $\mathrm{R}_{\mathrm{s}}(\Omega)$ & $\mathrm{R}_{\mathrm{ct}}(\Omega)$ & $\mathrm{Z}_{\mathrm{w}}\left(\mathrm{Ohm}_{\mathrm{s}} \mathrm{s}^{\wedge-1 / 2}\right)$ \\
\hline 1 & 1.7 & 105.3 & 278.0 & 45.1 \\
2 & 1.4 & 108.8 & 261.6 & 72.5 \\
3 & 1.5 & 110.1 & 253.4 & 79.5 \\
4 & 1.9 & 117.8 & 230.9 & 44.1 \\
5 & 1.6 & 123.4 & 202.8 & 53.2 \\
6 & 1.3 & 127.3 & 191.6 & 146.1 \\
7 & 2.0 & 131.5 & 182.5 & 219.2 \\
8 & 1.3 & 143.4 & 166.5 & 166.2 \\
9 & 1.4 & 159.9 & 145.3 & 85.9 \\
10 & 1.1 & 165.8 & 130.6 & 148.4 \\
50 & 1.5 & 167.6 & 110.8 & 143.5 \\
100 & 1.6 & 142.3 & 134.1 & 151.0 \\
500 & 1.4 & 160.1 & 112.3 & 97.8 \\
& & & & \\
\hline
\end{tabular}




\section{References}

1. Pan, E.; Jin, Y.; Zhao, C.; Jia, M.; Chang, Q.; Jia, M.; Wang, L.; He, X., Conformal Hollow Carbon Sphere Coated on $\mathrm{Sn}_{4} \mathrm{P}_{3}$ Microspheres as High-Rate and Cycle-Stable Anode Materials with Superior Sodium Storage Capability. ACS Appl. Energy Mater. 2019, 2, 1756-1764.

2. Zhang, J.; Wang, W.; Li, B., Effect of Particle Size on the Sodium Storage Performance of $\mathrm{Sn}_{4} \mathrm{P}_{3}$. J. Alloys Compd. 2019, 771, 204-208.

3. Choi, J.; Kim, W.-S.; Kim, K.-H.; Hong, S.-H., $\mathrm{Sn}_{4} \mathrm{P}_{3}-\mathrm{C}$ Nanospheres as High Capacitive and Ultra-Stable Anodes for Sodium Ion and Lithium Ion Batteries. $J$. Mater. Chem. A 2018, 6, 17437-17443.

4. Li, Q.; Li, Z.; Zhang, Z.; Li, C.; Ma, J.; Wang, C.; Ge, X.; Dong, S.; Yin, L., LowTemperature Solution-Based Phosphorization Reaction Route to $\mathrm{Sn}_{4} \mathrm{P}_{3} /$ Reduced Graphene Oxide Nanohybrids as Anodes for Sodium Ion Batteries. Adv. Energy Mater. 2016, 6, 1600376-1600386.

5. $\quad$ Fan, X.; Gao, T.; Luo, C.; Wang, F.; Hu, J.; Wang, C., Superior Reversible Tin Phosphide-Carbon Spheres for Sodium Ion Battery Anode. Nano Energy 2017, 38, 350-357.

6. Liu, J.; Kopold, P.; Wu, C.; van Aken, P. A.; Maier, J.; Yu, Y., Uniform Yolk-Shell $\mathrm{Sn}_{4} \mathrm{P}_{3} @ \mathrm{C}$ Nanospheres as High-Capacity and Cycle-Stable Anode Materials for Sodium-Ion Batteries. Energy Environ. Sci. 2015, 8, 3531-3538.

7. Usui, H.; Domi, Y.; Fujiwara, K.; Shimizu, M.; Yamamoto, T.; Nohira, T.; Hagiwara, R.; Sakaguchi, H., Charge-Discharge Properties of a $\mathrm{Sn}_{4} \mathrm{P}_{3}$ Negative Electrode in Ionic Liquid Electrolyte for Na-Ion Batteries. ACS Energy Lett. 2017, 2, 1139-1143.

8. $\quad$ Kim, Y.; Kim, Y.; Choi, A.; Woo, S.; Mok, D.; Choi, N.-S.; Jung, Y. S.; Ryu, J. H.; Oh, S. M.; Lee, K. T., Tin Phosphide as a Promising Anode Material for Na-Ion Batteries. Adv. Mater. 2014, 26, 4139-4144.

9. Pan, E.; Jin, Y.; Zhao, C.; Jia, M.; Chang, Q.; Zhang, R.; Jia, M., Mesoporous $\mathrm{Sn}_{4} \mathrm{P}_{3^{-}}$ Graphene Aerogel Composite as a High-Performance Anode in Sodium Ion Batteries. Appl. Surf. Sci. 2019, 475, 12-19. 